\title{
A PATHOLOGICAL STUDY OF FIVE CASES OF PYELONEPHRITIS IN THE NEWBORN
}

\author{
BY \\ K. A. PORTER and H. McC. GILES \\ From the Departments of Pathology and Paediatrics, St. Mary's Hospital, London
}

(RECEIVED FOR PUBLICATION MARCH 27, 1956)

It is increasingly recognized that many problems concerning the aetiology, recognition and natural history of urinary tract infections are still unresolved, and this is particularly true of such infections in infancy. There is, for example, no general agreement about their incidence in the first months of life: Stansfeld (1954) has pointed out that pyelonephritis occurs more frequently in the first year than at any other age, and most frequently of all in the first month, but this distribution pattern has not been universally recognized. The divergence of opinion may derive from a failure to consider pyelonephritis as a possible cause of such symptoms as vomiting, anorexia, constipation, diarrhoea and poor weight gain, or from uncertainty in assessing the significance of those minor abnormalities in the urine which may be the only diagnostic feature of chronic pyelonephritis. The situation is further confused by disagreement over the interpretation of various lesions which may be found in the kidneys of small infants dying of renal disease so that even necropsy may not resolve clinical uncertainty.

Among the few existing reports of the morbid anatomical findings in cases of neonatal pyelonephritis are those of Chown (1927) who could find only nine cases in the earlier literature. To these he added detailed reports of $\mathbf{3 0}$ cases of 'pyelitis' in infancy. Most of his cases showed the changes of acute suppurative pyelonephritis but four with chronic pyuria showed in addition 'inflammatory lesions in various stages of healing'. Craig (1935) gave the post-mortem findings in six cases of acute pyelonephritis and Hunt (1936) described one case with unilateral acute suppurative pyelonephritis fatal 13 days after birth. More recently Claireaux and Pearson (1955) have recorded chronic pyelonephritis in a newborn infant.

The histological picture of acute pyelonephritis in early infancy is indistinguishable from that found at any other age; in the chronic stage, however, differences may appear which have hitherto been insufficiently recognized and it is the purpose of this paper to discuss them.

\section{Material}

Five cases of pyelonephritis in infants under 1 month of age are described. All of these were males and this is in keeping with the general tendency of neonatal pyelonephritis to be more frequent in boys. Four of the infants were born in the same hospital within a few months of each other. (Two other cases occurring in the same period have apparently recovered.) The fifth case was a brother of one of the other four. One infant died during the acute phase of his illness, two died later in uraemia and two succumbed to intercurrent infection.

Case 1. R.E., a male negro infant, was the second of twins born on March 25, 1954, after a normal pregnancy. He was a breech presentation, but was turned and extracted without difficulty. It is not known whether the twins were identical or fraternal. He progressed normally until April 12 when he was noticed to be jaundiced. Haemoglobin was $8 \cdot 3 \mathrm{~g}$. \% and total serum bilirubin $7.4 \mathrm{mg} . \%$; the direct Coombs test was negative and no sickling was demonstrable. On April 16 he became lethargic, anorexic and febrile, and was given terramycin. The white blood count was $35,000 / \mathrm{c} . \mathrm{mm}$. and blood culture yielded a growth of $E$. coli : the urine contained pus and also yielded $E$. coli. The organisms obtained from the blood and urine appeared identical except that the former was sensitive and the latter resistant to terramycin. In spite of vigorous treatment with penicillin and streptomycin he died on April 18, aged $3 \frac{1}{2}$ weeks.

General Necropsy Findings. The tissues were dehydrated and bile-stained.

The myocardium was pale and flabby and the ductus arteriosus and foramen ovale were widely patent. The lungs showed a few areas of collapse at the right base.

The stomach and small gut were distended and 
contained white curdy material. The liver was deeply jaundiced, firm and without obvious pattern. There was a congenital stenosis of the common bile duct.

The left kidney (weight $25 \mathrm{~g}$.) was considerably larger than the right (weight $17 \cdot 5$ g.) and showed extensive acute pyelonephritis. Inflammation was much less marked in the right kidney. No other abnormality was seen in the genito-urinary tract.

On section of the brain a large haemorrhage was seen in the region of the right caudate nucleus which communicated with blood in the ventricles.

Microscopy of THE KIDNEYs. The renal capsule was normal. Scattered throughout both the cortex and medulla were multiple abscesses in which bacteria could be demonstrated. In these areas some of the glomeruli had been destroyed: others were extensively infiltrated by polymorphs or showed periglomerulitis with fibrin. Many of the tubules were dilated and contained pus. Polymorphs and some lymphocytes had infiltrated the interstitial connective tissue. The blood vessels were normal. The renal pelvis showed acute inflammation with areas of necrosis and loss of lining epithelium.

Microdissection showed a number of the nephrons to be of premature type in that they were shorter than normal and lacked convolutions.

Case 2. C.T. was a male infant born at term on March 4, 1950, by spontaneous, normal delivery. He had a weak cry with stridorous breathing, and laryngoscopy was thought to show a fine web across the vocal cords. Progress was slow with increasing reluctance to feed: on May 18 he was febrile and the urine contained pus and was found to be infected with $E$. coli and Str. faecalis. The infection did not clear in spite of treatment and he became increasingly acidotic and azotaemic. He died on July 20, 1950, aged 20 weeks.

General NeCropsy Findings. The body was slightly wasted with pale skin but no oedema. No congenital abnormality was found on external examination.

The heart weighed $40 \mathrm{~g}$. and showed left ventricular hypertrophy. In the larynx, situated immediately above the vocal cords which did not seem to be well developed, there was a very thin, triangular, transparent membrane. All lobes of both lungs showed moderate congestion and oedema and patchy areas of collapse. The intestines, liver, pancreas, spleen, pituitary, thyroid and adrenals were normal.

Each kidney weighed $20 \mathrm{~g}$. and the capsules stripped easily leaving a smooth pale surface. On section pallor was pronounced, the cortico-medullary junctions blurred and the pelves injected. The renal vessels and the remainder of the urogenital system were normal.

The brain and bones were normal.

Microscopy OF KIDNEYs. The capsule of the kidneys showed no change. The glomeruli were reduced to about three-quarters of their normal number. Of those surviving, most were normal but some showed marked pericapsular fibrosis. A few showed capsular crescent formation: the majority of the crescents had undergone fibrosis (Fig. 1) but some remained cellular. Adhesions between glomerular tuft and capsule were also to be seen

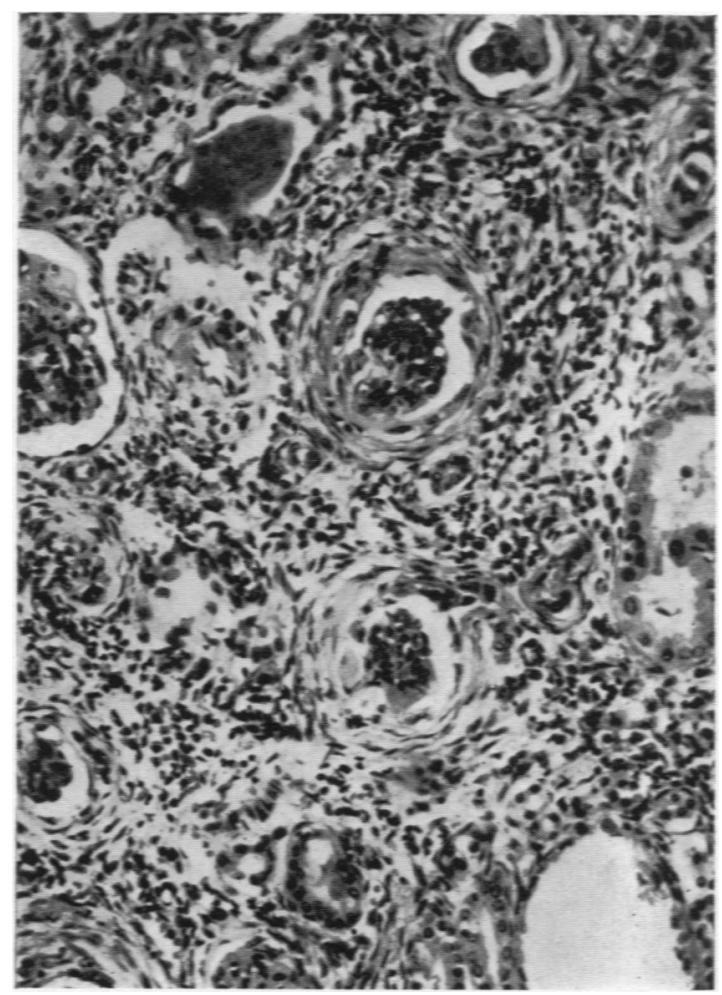

Frg. 1.-Case 2. Two glomeruli showing capsular crescents which have become fibrous. Note the dense interstitial infiltration with chronic inflammatory cells. (Haematoxylin and cosin $\times 150$ ).

in a few. Occasional glomeruli were partly hyalinized, others had been converted into hyaline spheres. The damaged glomeruli tended to be grouped together into rough cortical wedges. There were no striking changes in the glomerular capillary basement membranes. 'Alterative' glomerulitis was seen in some glomeruli in unscarred areas.

In the areas of glomerular damage groups of proximal tubules were dilated and lined by flattened atrophic epithelium and contained pink 'colloid' casts. Occasional tubules were cystic. Some collecting tubules showed similar changes.

The interstitial tissue, particularly in the cortical wedges of glomerular and tubular damage, was heavily infiltrated by lymphocytes and plasma cells with very occasional polymorphonuclear leucocytes. There was also some fibrosis.

The arcuate and interlobular arteries showed mild productive endarteritis and the arterioles mild hyperplastic arteriolosclerosis.

The pelvis of the kidney appeared normal.

Nephron dissection (Fig. 2) showed that the proximal tubule was considerably shorter than normal and almost completely devoid of convolutions: in addition the epithelium was flattened and the lumen grossly dilated (Darmady and Stranack, 1955). 


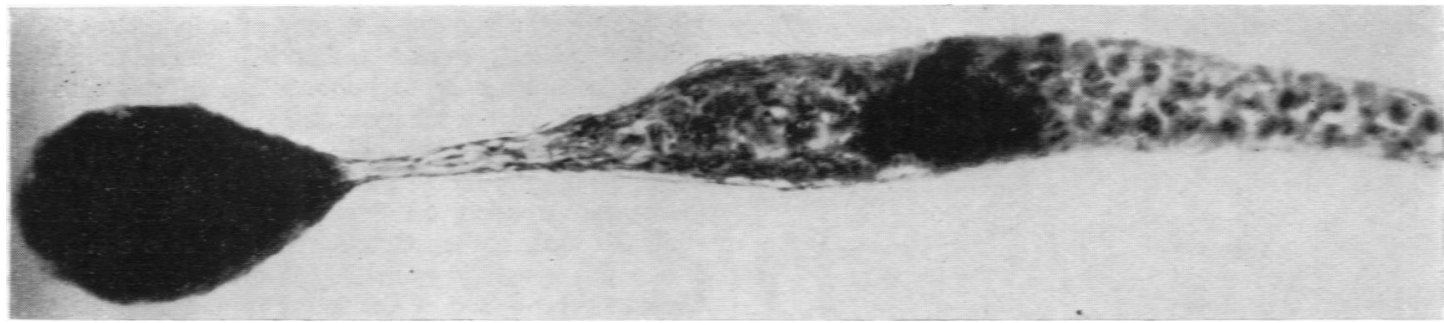

FIG. 2. Case 2. Microdissected nephron showing a short, straight grossly dilated proximal tubule with no convolutions and lined by flattened epithelium. (Phase contrast $\times 220$.)

Case 3. A.T., a brother of Case 2, was born at term on June 26, 1954, by spontaneous normal delivery. He had a stridorous cry which led his mother to recall his brother's history, and on July 1 his urine was therefore examined. It contained scanty pus cells, a moderate number of red blood cells and gave a heavy growth of $E$. coli. On July 7 the blood urea was $40 \mathrm{mg}$. ${ }^{\circ}$. In spite of intensive antibiotic treatment urine cultures continued to yield various organisms including $E$. coli, enterococci and Staph. aureus. The urine also repeatedly contained pus cells, red blood cells and hyaline casts, albumin in considerable amounts, and reducing substances. On July 13 the blood urea level was $132 \mathrm{mg} .{ }^{\circ}$ and remained high until death. On September 9 his systolic blood pressure was $150 \mathrm{~mm}$. He became increasingly acidotic, developed heart failure, and died on November 5, 1954, aged 19 weeks.

General NeCropsy Findings. The body showed oedema of the legs, scrotum, sacral region and eyelids. The abdomen was distended and the buttocks excoriated.

The heart weighed $50 \mathrm{~g}$. and showed great hypertrophy of the left ventricle, the wall being $1 \mathrm{~cm}$. in thickness. Each pleural cavity contained about $10 \mathrm{ml}$. of clear yellow fluid. The lungs were oedematous and showed patchy, blue, sunken areas of collapse. The larynx and trachea were normal. There was a small peritoneal effusion of clear yellow fluid. The liver was congested and enlarged, weighing $237 \mathrm{~g}$. The intestines, pancreas, spleen, pituitary, thyroid and adrenals were normal.

Each kidney weighed $20 \mathrm{~g}$. and the capsules stripped easily, leaving a smooth pale surface showing foetal lobation. The cortico-medullary junction was blurred. The pelvis was normal. The remainder of the genitourinary tract was normal.

The brain was oedematous. Bones were normal.

Microscopy OF KIDNEYs. The kidney capsule showed little change. The glomeruli were reduced to about half their normal number. Of the remainder few were completely normal and there was a tendency for the damaged glomeruli to be huddled together. Some showed marked concentric pericapsular fibrosis; in these the tuft was often relatively normal, but on other occasions tuft and capsule had fused to form a hyaline mass. In some there was proliferation of the cells of Bowman's capsule with crescent formation (Fig. 3). A few mitoses were visible in these cell masses and occasional capsular spaces had been obliterated. In a few cases the crescents had undergone fibrosis. Adhesions between tuft and capsule were frequent. Examples of pseudo-crescent formation (Claireaux and Pearson, 1955) and partly hyaline tufts were also seen. The capillary basement membranes of some of the affected glomerular tufts were thickened in a patchy manner and occasional tufts contained small droplets of fat apparently deposited between the capillary loops. In a few instances the capsular space contained acidophilic material but there were no polymorphs.

Most of the proximal tubules were dilated and contained eosinophilic, P.A.S.-positive colloid casts. The lining epithelium was flattened, with no brush border,

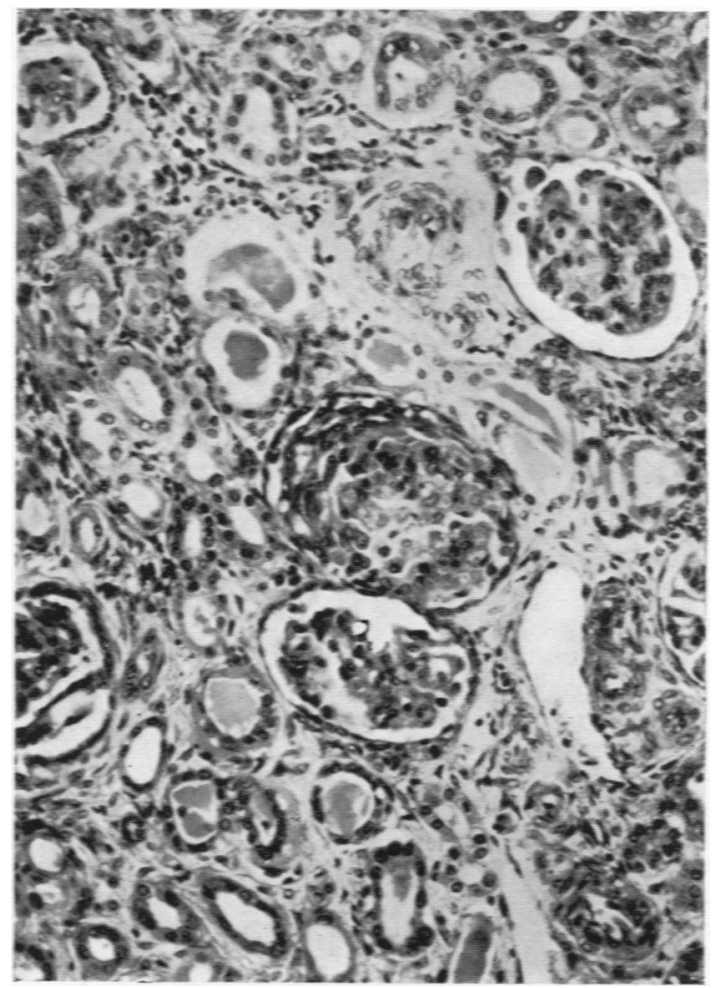

Fig. 3. Crescent composed of proliferating capsular epithelial cells. (Haematoxylin and eosin $\times 150$.) 


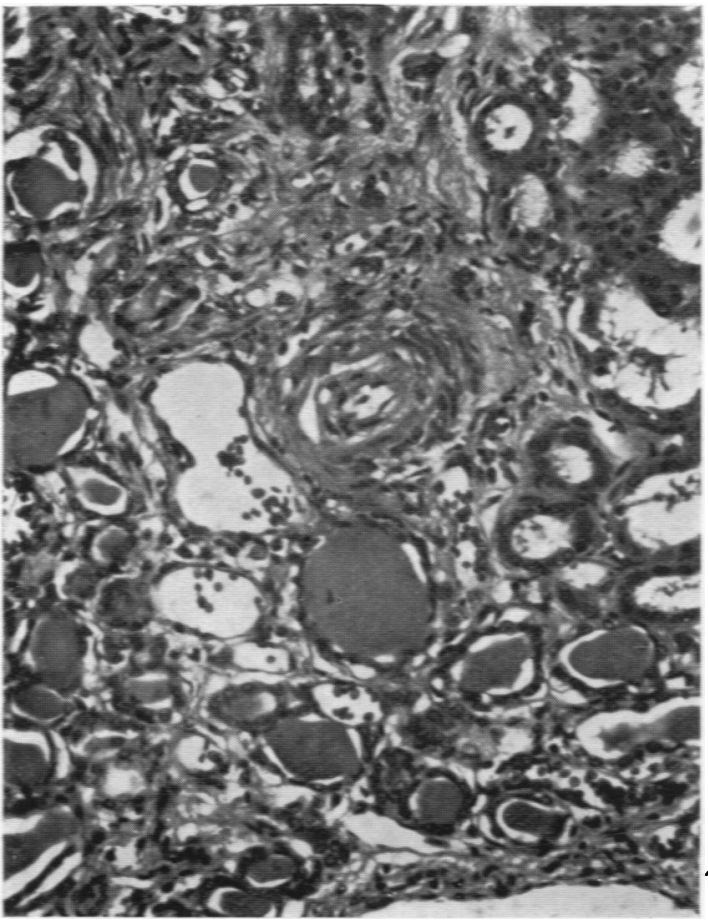

Fig. 4. Case 3. Numerous dilated tubules lined by flattened epithelium and containing 'colloid' casts. The artery shows productive endarteritis. (Hacmatoxylin and $\operatorname{cosin} \times 160$ ).

and lacked alkaline phosphatase. Many of the loops, distal tubules and collecting tubules contained pink casts, some of which were calcified. A few contained pus.

Large areas of interstitial tissue, particularly in the cortex, were densely infiltrated by plasma cells, lymphocytes, occasional eosinophils and histiocytes. In the medulla the chronic inflammatory infiltrate was much lighter. Interstitial fibrosis was also marked.

The arcuate and interlobular arteries showed marked productive endarteritis (Fig. 4) whilst many arterioles showed hyperplastic arteriolosclerosis and occasional fatty-hyaline change.

The epithelium of the renal pelvis was normal.

Nephron dissection showed the tubules to be essentially the same as those in the patient's brother (Case 2).

No organisms, inclusion bodies, cystine crystals or extramedullary haemopoiesis were found in this kidney.

Hyperplastic arteriolosclerosis was also found in the arterioles of the gut wall.

Case 4. S.R. was a boy born at term on August 25, 1954, by spontaneous normal delivery. On September 5 he began vomiting and on September 9 the urine contained many pus cells and yielded a mixed growth of micrococci and proteus. On the same day blood culture yielded a growth of $E$. coli and four days later an apparently identical coliform organism was cultured from the urine. On September 15 the blood urea level was
$100 \mathrm{mg} . \%$ and the systolic blood pressure was $80 \mathrm{~mm}$. He was given a prolonged course of sulphonamide and chloramphenicol, during which his general condition improved and his urine became free of pus cells but continued to yield a scanty mixed growth of enterococci, micrococci, proteus and coliforms. Blood urea fell to about $50 \mathrm{mg}$. \% but did not return to normal. Treatment was stopped on September 28. He remained clinically well, but on October 21 the urine again contained pus cells and grew $E$. coli and Proteus, and the haemoglobin was $8.9 \mathrm{~g}$. \%. From October 25 to November 11 he was given sulphonamide, penicillin and streptomycin, in spite of which the urine continued to show a small excess of pus cells and a mixed growth of organisms. The blood urea level remained at 50 to $60 \mathrm{mg} . \%$. His general progress was satisfactory until January 12, 1955, when he developed a slight cough: on January 15 his mother found him dead in his cot. At death he was aged 21 weeks.

General NeCropsy Findings. The body was well nourished, showing cyanosis of the lips and finger nails and bile-stained fluid coming from the mouth.

The heart weighed $39 \mathrm{~g}$. and showed left ventricular hypertrophy, moderate dilatation of the right side, and a few petechiae beneath the epicardium. There was frothy mucopus in the trachea, main bronchi and many of the smaller bronchi, particularly in the left lower lobe, with considerable injection of the lining mucosa. The lungs showed extensive, patchy, lobular collapse, most marked in the lower lobes, and generalized oedema. Microscopically there was a purulent bronchiolitis. The hilar lymph nodes were enlarged and haemorrhagic. The liver was enlarged and congested. The gastro-intestinal tract, pancreas, spleen, adrenals, thyroid, thymus and pituitary were normal.

The kidneys (weight $31 \mathrm{~g}$. and 27 g.) were congested. The capsules stripped readily, exposing surfaces with a fine irregular granularity. No abnormality was seen on section. The pelves, renal vessels and the remainder of the urogenital system were normal.

The brain and bones were normal.

Microscopy OF THE KiDNEYS. The renal capsule was normal. The glomeruli were slightly reduced in number. Most of the surviving glomeruli were normal, but in a roughly wedge-shaped area; they were closer together than usual and showed various changes, the commonest of which was marked concentric pericapsular fibrosis, often with relatively normal tufts. However, in some this had led to complete obliteration of the tufts so that only a hyaline sphere remained. Some showed adhesions between tuft and capsule. Occasional crescents were seen (Fig. 5) and a few primitive glomeruli.

In the patches where the glomeruli were affected some of the proximal tubules appeared atrophic with flattened epithelium. However, colloid casts were few, and dilatation was not a feature. The distal parts of the tubules seemed normal. Some primitive tubules were also seen.

Fibrosis and chronic inflammatory infiltration of the interstitium with lymphocytes, plasma cells and occasional eosinophils were features of the scarred areas.

The arcuate and interlobular arteries showed slight 


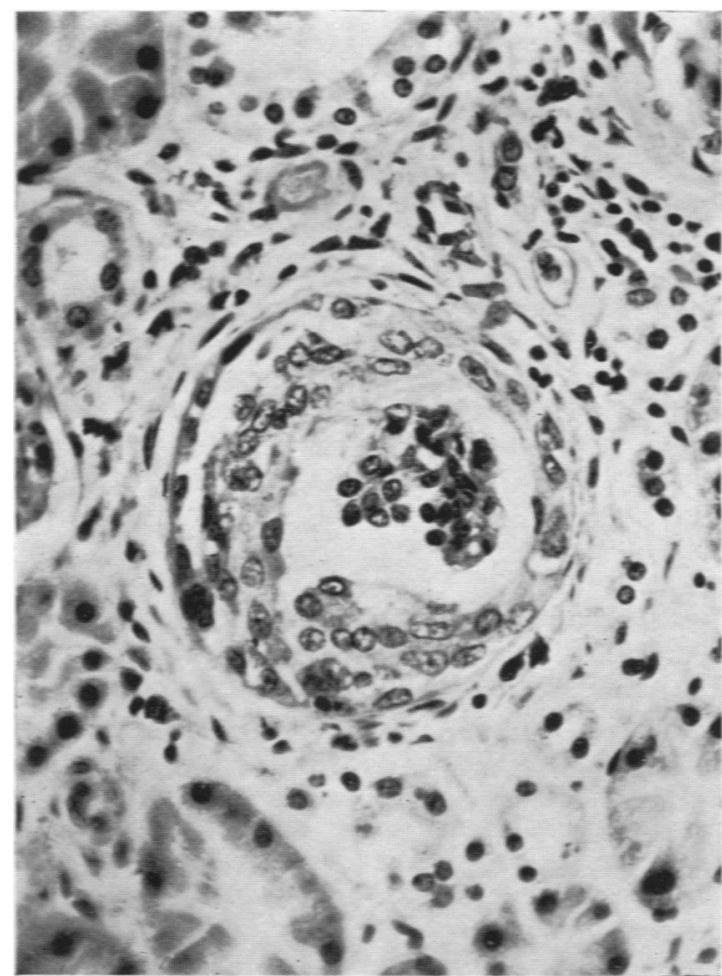

Frg. 5. Case 4. Glomerulus with a well defined cellular capsular crescent. (Haematoxylin and cosin $\times 350$.)

productive endarteritis and the arterioles mild hyperplastic arteriolosclerosis.

The epithelium of the renal pelvis was normal.

Microdissection showed little evidence of persisting foetal nephrons. However, there were a number of long loops of Henle.

Arteries and arterioles in other organs were normal.

Case 5. R.F. was a male infant born at term on May 23, 1954, by spontaneous normal delivery. He made normal progress until June 5 when he refused his feeds and was noticed to be jaundiced. The liver was enlarged and the tip of the spleen and left kidney were palpable. On June 6 the urine contained pus cells and yielded a growth of $E$. coli. On June 9, the blood urea level was $160 \mathrm{mg}$. ${ }^{\circ}{ }_{0}$. Between June 7 and July 6 he was treated with sulphonamide, penicillin, streptomycin, terramycin, chloramphenicol and erythromycin in various combinations. From mid-June the jaundice faded: whether it had been due to infection or to neonatal hepatitis was uncertain. His general condition improved slowly and irregularly and although the urine became free of pus cells it continued to yield a growth of proteus, coliforms and micrococci. By July 10 he was thought to be clinically well, and on July 28 he was discharged from hospital. He did not return for follow-up but was believed by his parents to be progressing satisfactorily until September 3 when he developed a severe attack of vomiting and diarrhoea. He was admitted to hospital but died on September 4, aged $14 \frac{1}{2}$ weeks.

General NeCropsy Findings. The body was severely dehydrated. The heart weighed $40 \mathrm{~g}$. and showed left ventricular hypertrophy. There was bilateral basal pulmonary congestion. The tongue was flabby and furred and there was hyperaemia of the mucous membranes of the stomach and small intestine suggestive of an acute gastro-enteritis. The liver and spleen were a little enlarged. The brain and bones were normal.

The kidneys (weight $27 \mathrm{~g}$. and $24 \mathrm{~g}$.) appeared normal and their capsules stripped easily exposing smooth subcapsular surfaces. No abnormalities were seen in the remainder of the urogenital system.

Microscopy of KIDNeys. The renal capsule was normal. The glomeruli were reduced to about threequarters of their normal number. Many showed pericapsular fibrosis with relatively normal tufts, some showed adhesions between tuft and capsule, others well marked capsular crescents. Some glomeruli had become completely hyalinized. There damaged glomeruli tended to be huddled together in rough cortical wedges. Unaffected glomeruli had undergone compensatory hypertrophy.

Many of the proximal tubules were dilated and some contained 'colloid' casts. Some of their lining epithelial cells were atrophic, others showed hyaline droplet degeneration. Distal and collecting tubules appeared normal. There were occasional casts in the collecting tubules, some showing calcification with tubular syncytial cell formation around them.

There was increased medullary interstitial and perivascular fibrous tissue, with occasional foci of calcification in cortex and medulla and a scattered infiltration by lymphocytes, plasma cells and occasional eosinophils and neutrophils.

The arcuate and interlobular arteries showed mild productive endarteritis, whilst the walls of occasional arterioles showed hyperplastic arteriolosclerosis.

The renal pelvis was lightly infiltrated by chronic inflammatory cells and subepithelial fibrosis was prominent.

These kidneys were not microdissected.

Arteries and arterioles in other organs were unaffected.

\section{Discussion}

Morphological Differences from Pyelonephritis in Adults. Case 1 died after an acute illness of relatively brief duration. The kidneys showed the typical histological picture of acute pyelonephritis, differing in no way from that seen in the older child or adult. In the remaining cases, there was a more prolonged illness, during which nitrogen retention and persistent pus cells and organisms in the urine led to the clinical diagnosis of chronic pyelonephritis. The histology of the kidneys in all four cases is believed to support this diagnosis, satisfying as it does the criteria laid down by Weiss 
and Parker (1939), namely, (1) marked infiltration of the interstitial tissue with lymphocytes, plasma cells and eosinophils; (2) presence of pericapsular fibrosis; (3) presence of so-called colloid casts in tubules lined by atrophic epithelium.

The only other condition with which these histological changes might be confused is glomerulonephritis. In the present cases, however, many glomeruli were unaffected, whereas in glomerulonephritis it is usual for the changes to be diffuse with involvement of almost all glomeruli. Moreover, in those glomeruli in which pericapsular fibrosis was present, the tufts were often normal; in glomerulonephritis there is usually a fairly close correlation between the degree of fibrosis of the tufts and of Bowman's capsule. In glomerulonephritis the interval between glomeruli is diminished but these distances tend to be fairly uniform, in sharp contrast to the rather wedge-shaped clusters of glomeruli we have described. Lastly the interstitial infiltration with chronic inflammatory cells was greater in the present cases than is usual with glomerulonephritis.

However, the kidneys in these cases differed in two respects from those found in adults suffering from chronic pyelonephritis: (1) Macroscopically, there was little asymmetrical contraction or coarse, shallow scarring. (2) Microscopically, crescents in varying numbers were prominent.

Similar observations were made by Claireaux and Pearson (1955) in their case of chronic pyelonephritis in a newborn infant.

The absence of scarring may be a consequence of the comparatively short period of time for which the pyelonephritic process had been in progress, since all the cases were aged 21 weeks or under at the time of death. The presence of crescents does not necessarily exclude the diagnosis of chronic pyelonephritis. Crescents are not specific for glomerulonephritis, inasmuch as identical formations have long been known to occur in cases of malignant nephrosclerosis, subacute bacterial endocarditis, and sometimes in the chronic pyelonephritis of adults. In these latter conditions, however, the crescents usually involve only occasional glomeruli, not the majority as in glomerulonephritis.

It is possible that epithelial crescents develop in response to various stimuli more readily in the newborn period. Lowe, Henderson, Park and McGreal (1954) described crescent formation in a renal biopsy from a child of 1 year 9 months suffering from the severe form of idiopathic hypercalcaemia with low-grade secondary chronic pyelonephritis. Campbell and Henderson (1949), discussing asymmetrical cortical necrosis of kidneys in infancy, mentioned crescents in their Case 3, and Friedman, Grayzel and Lederer (1942) described a proliferation of the parietal layer of Bowman's capsule, giving a crescent-like appearance, as an occasional finding in infants of average age 2.7 months in the absence of evidence of any inflammatory reaction in the kidneys.

If it be accepted that crescents do not necessarily mean glomerulonephritis, then, as suggested by Claireaux and Pearson (1955), many cases described previously as neonatal glomerulonephritis may well have been cases of chronic pyelonephritis. Recent examples are the cases described by Thompson (1951), Collins (1954) and Kunstadter and Rosenblum (1954).

Possible Role of Congenital Abnormalities of the Nephron. Once past the neonatal period, boys seldom develop pyelonephritis except as a complication of some manifest urinary tract anomaly. All the infants in the present series were males but none of them showed any such anomaly. The possibility arises that a defect of the nephron, presumably congenital and demonstrable only microscopically, might be the predisposing lesion in these infants. The assessment of this possibility is complicated by the fact that kidneys from young infants dying of various diseases frequently show glomeruli and tubules which are 'sclerosed', immature or otherwise atypical. The cause and significance of these abnormalities is not clear. Friedman et al. (1942) have given the name 'congenital glomerulosclerosis' to the hyaline changes in the glomerular tufts and Bowman's capsule which were first described by Herxheimer in 1908. Friedman found these lesions in 12 out of 100 consecutive infants dying under the age of 14 months; he pointed out their relationship to obliterative changes in the corresponding afferent arteriole, but could advance no more fundamental explanation for their occurrence. It is interesting that the lesions were found about three times as often in males as in females, this being the estimated ratio of pyelonephritis in males to females in the first few months of life.

Marshall (1953), in a series of kidneys from infants and children, described structures which, on morphological grounds, he believed to be persisting foetal glomeruli and tubules. In most of the older infants and children pyelonephritis was present and Marshall suggested that such infections had as their underlying lesion a focal or localized dysplasia of the renal parenchyma.

In four cases of the present series (Nos. 1, 2, 3 and 4), Dr. E. M. Darmady and Miss Fay Stranack have shown by painstaking microdissection of the 
kidneys that the nephrons were abnormal. In Cases 1, 2 and 3 the findings were reminiscent of the state of affairs in the immature nephrons seen in the kidneys of premature babies. It is to be noted that two patients in whom these atypical nephrons were found were brothers; this provides circumstantial evidence for the view that the lesions are congenital but at the same time makes it unjustifiable to draw conclusions from what may be a special case about the relationship of such lesions to neonatal pyelonephritis in general. Nevertheless, it remains an attractive hypothesis that these or other not easily recognized congenital abnormalities of the nephron may play an important role in predisposing certain babies to renal infection.

The Outcome of Neonatal Pyelonephritis. The majority of infants who develop an attack of acute pyelonephritis apparently recover. In a few cases, however, the renal disease may smoulder on, ultimately to cause hypertension, bone lesions, renal failure, or a combination of these. The proportion of cases in which this happens is unknown, and may be higher than is generally suspected. All four cases of chronic pyelonephritis in the present series showed left ventricular hypertrophy of varying degree and renal arteriolar lesions. This suggests that these cases were probably hypertensive, although only in Case 3 was hypertension actually demonstrated during life. The histological changes found in the kidneys closely resemble those described in many cases of 'renal rickets'. This lends support to the view expressed by Weiss and Parker (1939) that the great majority of infants and children who show so-called chronic interstitial nephritis associated with dwarfism and bone changes are in reality examples of long-standing chronic pyelonephritis.

In the light of these observations we would reinforce Stansfeld's plea for the consideration of pyelonephritis as a possible diagnosis in any infant who vomits, loses weight or goes off his food. Furthermore, we would urge that known cases of pyelonephritis should be followed up with the utmost care, and that apparently minor abnormalities of their urine should not be too readily disregarded.

\section{Summary}

Five cases of neonatal pyelonephritis in male infants are described. All the babies died before they were 5 months old and the necropsy findings are reported.

The kidneys in four cases showed changes believed to be those of chronic pyelonephritis. The appearances differed from those usually found in older children and adults with chronic pyelonephritis in that asymmetrical contraction and coarse shallow scarring were absent and crescents in varying numbers were prominent.

Microdissection of the kidneys from four patients, two of whom were brothers, showed abnormalities of the nephron.

We are grateful to Dr. R. Lightwood, Dr. T. Pearse Williams and Dr. T. Stapleton for permission to report on patients under their care; to Dr. J. L. HamiltonPaterson, Dr. E. D. Hoare and Dr. P. L. Masters for permission to use material from Cases 2 and 4 ; to Dr. E. M. Darmady and Miss Fay Stranack for kindly undertaking the kidney microdissections; and to Professor Pryce and Dr. R. Lightwood for help with the manuscript.

We are grateful to the Hon. Editors of the Proceedings of the Royal Society of Medicine for permission to publish Fig. 4.

\section{REFERENCES}

Campbell, A. C. and Henderson, J. L. (1949). Archives of Disease in Childhood, 24, 269

Chown, B. (1927). Ibid., 2, 97

Claireaux, A. E. and Pearson, M. G. (1955). I Ibid., 30, 366.

Collins, R. D. (1954). A.M.A. Amer. J. Dis. Child., 87, 478.

Craig, W. S. (1935). Archives of Disease in Childhood, 10, 337

Darmady, E. M. and Stranack, F. (1955). Proc. roy. Soc. Med., 48, 781.

Friedman, H. H., Grayzel, D. M. and Lederer, M. (1942). Amer. J. Path., 18, 699.

Herxheimer, G. (1908). Frankfurt Z. Path., 2, 138.

Hunt, J. S. (1936). Arch. Pediat., 53, 341.

Kunstadter, R. H. and Rosenblum, L. (1954). A.M.A. Amer.J. Dis. Child., 88, 611 .

Lowe, K. G., Henderson, J. L., Park, W. W. and McGreal, D. A. (1954). Lancet, 2, 101

Marshall, A. G. (1953). Brit. J. Surg., 41, 38.

Stansfeld, J. M. (1954). Proc. roy. Soc. Med., 47, 631

Thompson, A. C. (1951). W. Va med. J., 47, 16.

Weiss, S. and Parker, F. (1939). Medicine (Baltimore), 18, 221. 